\title{
Polyelectrolyte adsorption onto oppositely charged interfaces: unified approach for plane, cylinder, and sphere
}

\author{
A. G. Cherstvy ${ }^{* a}$ and R. G. Winkler ${ }^{b}$ \\ Received 14th March 2011, Accepted 19th April 2011 \\ DOI: $10.1039 / \mathrm{c} 1 \mathrm{cp20749k}$
}

A universal description is presented for weak adsorption of flexible polyelectrolyte chains onto oppositely charged planar and curved surfaces. It is based on the WKB (Wentzel-Kramers-Brillouin) quantum mechanical method for the Green function equation in the ground state dominance limit. The approach provides a unified picture for the scaling behavior of the critical characteristics of polyelectrolyte adsorption and the thickness of the adsorbed polymer layer formed adjacent to the interface. We find, particularly at low-salt conditions, that curved convex surfaces necessitate much larger surface charge densities to trigger polyelectrolyte adsorption, as compared to a planar interface in the same solution. In addition, we demonstrate that the different surface geometries yield very distinct scaling laws for the critical surface charge density required to initiate chain adsorption. Namely, in the low-salt limit, the surface charge density scales cubical with the inverse Debye screening length for a plane, quadratic for an adsorbing cylinder, and linear for a sphere. As the radius of surface curvature grows, the parameter of critical chain adsorption onto a rod and a sphere turns asymptotically into that of a planar interface. The transition occurs when the radius of surface curvature becomes comparable to the Debye screening length. The general scaling trends derived appear to be consistent with the complex-formation experiments of polyelectrolyte chains with oppositely charged spherical and cylindrical micelles. Finally, the WKB results are compared with the existing theories of polyelectrolyte adsorption and future perspectives are outlined.

\section{Introduction}

Adsorption of polyelectrolyte (PE) chains onto oppositely charged surfaces has attracted considerable theoretical attention over the last decades ${ }^{1,2}$ because of its importance for many biological and technological applications. A number of theoretical approaches have been utilized, in particular for weak PE adsorption onto curved oppositely charged interfaces immersed in electrolyte solutions. Here, the properties of the adsorption process are basically dictated by a competition of attractive electrostatic PE-surface interactions and a loss of polymer conformational entropy upon adsorption, ${ }^{3,4}$ with the key parameters being the PE charge density, its persistence length, ionic strength of the solution, and PE-surface affinity. The adsorption of PEs onto curved surfaces is generally not amenable to exact solutions, in contrast to the planar case, and therefore a number of approximate approaches have been suggested in the literature. ${ }^{7-11}$

In particular, for adsorption of long PE chains onto spherical and cylindrical surfaces, the variational method based on trial

\footnotetext{
${ }^{a}$ Institute of Complex Systems, ICS-2, Forschungszentrum Jülich,

52425 Jülich, Germany.E-mail: a.cherstvy@gmail.com

${ }^{b}$ Institute for Advanced Simulation, IAS-2, Forschungszentrum Jülich, 52425 Jülich, Germany
}

functions has been implemented in ref. 12. Also, the adsorption of flexible PEs onto a spherical surface has been investigated in ref. 13-15 by approximating the Debye-Hückel PE-sphere attraction with the Hulthen potential that allows to determine an exact analytical solution. These results unravelled the scaling characteristics of PE adsorption, including the critical conditions for the adsorption-desorption transition and the thickness of the adsorbed PE layer. These two quantities are also the main targets of the present work.

A number of computer simulation studies on complexation of finite-length PE chains with oppositely charged spheres and rods have been published in recent years as well. ${ }^{16-21} \mathrm{~A}$ terse style of this paper does not allow us to discuss here the wealth of data and trends uncovered in simulations. Experimentally, the complex formation of various PE chains (both flexible and semi-flexible) with oppositely charged particles (micelles, dendrimers, and proteins) has been systematically investigated by the P. Dubin group. ${ }^{22-27}$ These studies provide valuable data for the onset on PE-particle complexation (detected via a subsequent precipitation of complexes from the solution) that can be associated with the conditions determined theoretically for the critical PE-surface adsorption. Despite these active theoretical and computational research activities, the universal scaling behavior for the critical PE adsorption conditions is 
still under debate for curved surfaces, partly because of the lack of a general methodology for different geometries.

In this letter, we bridge this gap and propose an approximate but reliable universal approach to treat PE adsorption onto planar, cylindrical, and spherical oppositely charged interfaces. The paper is organized as follows. In the next section, we review the basic equations for the Green function and discuss a set of approximations implemented in the description of conformational properties of PE chains adsorbed on a surface. Then, we summarize the general concepts of the WKB approach. In the subsequent section, utilizing the WKB approximation, we explore the physical properties of PE adsorption onto surfaces of various geometries. We compare and contrast the WKBscaling results with previously derived theoretical predictions for the thickness of the adsorbed PE layer and the critical adsorption characteristics (surface charge density, temperature, etc.). Interesting experimental observations on complexation of PEs with oppositely charged spherical and cylindrical objects are discussed at the end, in the light of scaling predictions and a salt-dependent PE electrostatic persistence length.

\section{Modelling PE adsorption: Green function and approximations}

The weakly charged flexible PE chain is described by a continuous space curve with the linear charge density $\rho=e_{0} / b_{0}$, where $e_{0}$ is the elementary charge and $b_{0}$ the inter-charge separation. The intra-molecular self-interactions (e.g. Coulomb repulsive and excluded-volume interactions) are not taken into account explicitly in the model, but they are rather accounted for via the value of the Kuhn segment length of the chain $b$. This length sets the length-scale in the problem. The excluded-volume effects are indeed important when relatively dense PE profiles are formed near the interface. For the critical adsorption conditions, when the PE chains are only weakly perturbed by the adsorbing interface, these effects are, however, expected to have a rather small impact, see Discussion section. The PE chains are weakly charged, below the Manning limit for counterion condensation.

The oppositely charged interface has a homogeneous surface charge density $\sigma$, being impenetrable to solvent molecules and PE chains. The cylinder and sphere have the radius $a$. The solvent is treated as a medium of constant dielectric permittivity, $\varepsilon=80$. The counterions and salt ions are taken into account on the level of the linearized Poisson-Boltzmann equation. Its solution yields the PE-surface interaction potential, i.e., the Debye-Hückel potential for the corresponding geometry. The latter is derived assuming a constant surface charge density.

The conformational properties of flexible Gaussian polymer chains of length $L(L \rightarrow \infty)$ and the average spatial distribution of its monomers follow from the probability density $G\left(\mathbf{r}, \mathbf{r}^{\prime} ; L\right)$, the Green function. Here $\mathbf{r}^{\prime}=\mathbf{r}(0)$ and $\mathbf{r}=\mathbf{r}(L)$ denote the positions of the polymer end points. For a PE chain in the electrostatic field of an attracting surface $V_{D H}(\mathbf{r})$ the Green function obeys the equation

$$
\left(\frac{\partial}{\partial L}-\frac{b}{6} \nabla_{\mathbf{r}}^{2}+\frac{V_{D H}(\mathbf{r})}{k_{\mathrm{B}} T}\right) G\left(\mathbf{r}, \mathbf{r}^{\prime} ; L\right)=\delta\left(\mathbf{r}-\mathbf{r}^{\prime}\right) \delta(L),
$$

with $k_{\mathrm{B}}$ the Boltzmann constant and $T$ the temperature. The Laplacian term $\nabla_{\mathbf{r}}^{2} G\left(\mathbf{r}, \mathbf{r}^{\prime} ; L\right)$ describes the conformational entropy of the chain. This equation has to be solved with the boundary conditions $G=0$ at the surface and far away from it. The first condition is attributable to an entropic repulsion of polymer chains from the immediate vicinity of the adsorbing interface. The second one prescribes the vanishing $\mathrm{PE}$ concentration in the bulk.

The standard procedure to find the solution of eqn (1) for long enough polymers is the eigenfunction expansion,

$$
G\left(\mathbf{r}, \mathbf{r}^{\prime} ; L\right)=\sum_{n} \psi_{n}^{*}\left(\mathbf{r}^{\prime}\right) \psi_{n}(\mathbf{r}) e^{\mu_{n} L} .
$$

In the ground state dominance approximation for a long chain (a dozen of Kuhn lengths), the lowest eigenvalue $\mu \equiv \mu_{0}$ gives the dominant contribution to the Green function $(\mu>0$ for the adsorbed state). The following equation applies for the eigenfunction in the ground state $\psi \equiv \psi_{0}$,

$$
\left(-\frac{b}{6} \nabla_{\mathbf{r}}^{2}+\frac{V_{D H}(\mathbf{r})}{k_{\mathrm{B}} T}\right) \psi(\mathbf{r})=-\mu \psi(\mathbf{r}) .
$$

Below, the WKB method is employed to solve this eigenvalue equation for the conditions of weak PE-surface adsorption.

The term "weak adsorption" here implies that the entropic free energy of PE chains is comparable to their electrostatic attraction energy to the interface. The chain is assumed to be Gaussian and its conformations are only weakly perturbed by interactions with the surface. This is the most severe approximation of the current model. It can be relaxed in several ways that is, however, beyond the scope of this study. We also assume that the PE profile is built up near the adsorbing surface without disturbing the electrostatic potential and ionic distribution near the interface prescribed by the PoissonBoltzmann theory. A more general self-consistent field theory, with a coupling of $\mathrm{PE}$ and ionic distributions next to the interface, has been presented in refs. 28 and 29. Certain polymer stiffness effects for PE adsorption onto curved surfaces due to a salt-dependent persistence length are discussed in the last section.

We use the adsorption parameter $\delta$, as defined previously in ref. 13, that couples the strength of electrostatic PE-surface attraction, absolute temperature, polymer persistence length $b / 2$, and the surface radius $a$. It can be written via the interaction parameter $\theta=4 \pi|\rho \sigma| /\left(\varepsilon k_{\mathrm{B}} T \kappa\right)$ as follows

$$
\delta=\frac{6 a^{3} \kappa \theta}{b}=\frac{24 \pi a^{3}|\rho \sigma|}{\varepsilon k_{\mathrm{B}} T b},
$$

where $\kappa$ is related to the Debye screening length $\lambda_{\mathrm{D}}$ as $\lambda_{\mathrm{D}}=1 / \kappa$. Strictly speaking, the conditions $a \gg b$ and $\lambda_{\mathrm{D}} \gg b_{0}$ have to be satisfied. For a fixed PE charge density and persistence length, PE adsorption takes place when the adsorption parameter becomes larger than some critical value, $\delta>\delta_{c}$. This corresponds to the formation of a first bound PE state that takes place, e.g., when the surface charge density exceeds some critical level, $\sigma>\sigma_{c}$. Finding the dependence of $\sigma_{c}$ on environmental and model parameters such as $\kappa, T, a, b$ is the basic goal of this study.

From eqn (4) we note right away that larger surface charge densities $\sigma_{c}$ are required to trigger the adsorption of stiffer 
polymers (larger $b$ values). Also, it is physically clear that in concentrated electrolyte solutions, where the PE-surface attraction is well-screened by mobile ions, again, larger surface charge densities and higher linear PE charge densities are necessary to stimulate a PE deposition onto the interface.

\section{WKB method: general procedure}

To solve eqn (3), we employ the WKB approach used in quantum mechanics to find approximate solutions of the time-independent Schrödinger equation..$^{30}$ The latter is equivalent to the Edwards equation for the polymer probability density. The WKB approach provides an approximate solution of the one-dimensional eigenfunction equation

$$
\psi(x)_{x x}+Q^{2}(x) \psi(x)=0,
$$

on both sides of a simple-zero point $x_{0}$ of the potential $Q^{2}\left(x_{0}\right)=0$. Here, $\psi_{x x}$ denotes the second derivative of $\psi$ with respect to $x$. The WKB method provides the solution of a more general equation

$$
\psi(x)_{x x}+\left[Q^{2}(x)-\bar{\theta}(x)\right] \psi(x)=0,
$$

in the form

$$
\psi(x)=S(x)\left[\alpha \xi^{1 / 3} J_{-1 / 3}(\xi)+\beta \xi^{1 / 3} J_{1 / 3}(\xi)\right],
$$

where $J_{ \pm 1 / 3}$ are the Bessel functions of fractional order ${ }^{31}$ and the supplementary functions are given by $\bar{\theta}(x)=S(x)_{x x} / S(x)$ and $S(x)=Q^{-1 / 2}(x) \xi^{1 / 6}(x)$. Here, the variable $\xi$ is defined as $\xi(x)=\int_{x_{0}}^{x} Q\left(x^{\prime}\right) \mathrm{d} x^{\prime}$ for $x>x_{0}$ in the region $Q^{2}>0$, whereas $\xi(x)=\int_{x}^{x_{0}}\left|Q\left(x^{\prime}\right)\right| \mathrm{d} x^{\prime}$ for $x<x_{0}$ in the region $Q^{2}<0$. If the condition $|\bar{\theta}(x)| \ll|Q(x)|$ is satisfied, the general solution of eqn (7) yields also an approximate solution of eqn (5).

The two eigenfunctions describe the oscillating $\left(J_{-1 / 3}\right)$ and decaying $\left(J_{1 / 3}\right)$ branches of the solution. They can be attributed in quantum-mechanical analogy to the wave function of a quantum particle confined in a potential well or penetrating into a potential barrier, respectively. Using the properties of Bessel functions, ${ }^{32} \psi(x)$ can be expressed in terms of Airy functions $A i$ of negative and positive arguments, respectively. Namely, using the definition $R(x)=-Q^{2}(x)$, one arrives at the uniformly valid Langer's solution that at $x>x_{0}$ has the form

$$
\psi \propto(-R)^{-1 / 4} \xi^{1 / 6} A i\left(-(3 \xi / 2)^{2 / 3}\right),
$$

and converges at infinity, whereas for $x<x_{0}$, with the definition $R(x)=Q^{2}(x)$, the solution is given by

$$
\psi \propto R^{-1 / 4} \xi^{1 / 6} \operatorname{Ai}\left((3 \xi / 2)^{2 / 3}\right) .
$$

For large arguments, the corresponding Airy functions can asymptotically be expanded in sinusoidal and exponentiallydecaying functions.

We confirmed that the WKB applicability condition $|\bar{\theta} / Q| \ll 1$ is satisfied for all three geometries. It works particularly well far from the adsorption-desorption transition, while it naturally fails in the proximity of the zero-potential point $x_{0}$ at which $Q\left(x_{0}\right)=0$. Note that the WKB technique has been applied to the analysis of weak PE-rod adsorption in ref. 10.

\section{Basic equations and results}

\section{a Plane}

The Edwards equation for the Green function describing the probability density of a charged flexible polymer in the vicinity of a planar interface is

$$
-\frac{b}{6} \psi(x)_{x x}-\theta e^{-\kappa x} \psi(x)=-\mu \psi(x) .
$$

It has already the form $\psi_{x x}-R \psi=0$ with the $\mathrm{WKB}$ potential

$$
R(x)=\frac{6}{b}\left(\mu-\frac{\delta b e^{-\kappa x}}{6 a^{3} \kappa}\right) .
$$

This potential can be either positive or negative depending upon the eigenvalue $\mu$, the strength of the PE-surface adsorption $\delta$, the separation from the surface, and salt conditions. The potential attains zero at $\kappa x_{0}=-\ln \left(6 a^{3} \kappa \mu /(\delta b)\right)$. For a plane, any dependence on $a$ vanishes because $\delta \propto a^{3}$, but the expressions below are presented in the form similar to the rod and sphere situations.

For the oscillating solution, realized near the interface at $0<x<x_{0}$, we have $R<0, \xi(x)=\int_{x}^{x_{0}} \sqrt{-R\left(x^{\prime}\right)} \mathrm{d} x^{\prime}$, and the
PE density profile is expressed as

$$
P(x) \propto \psi^{2} \propto\left\{\xi^{1 / 6}(-R)^{-1 / 4} A i\left(-(3 \xi / 2)^{2 / 3}\right)\right\}^{2} .
$$

The existence of the PE bound state near the surface is dictated by the properties of the attractive adsorption potential, mainly by its strength and decay length. Namely, the PE profile created close to the surface has to satisfy the condition $P(0)=0$ that involves the first zero of the Airy function, $a i_{1} \approx-2.338$. Thus, the first maximum/peak of the oscillating solution describes the adsorbed PE layer near the interface. This condition yields $\xi(0)=2\left|a i_{1}\right|^{3 / 2} / 3$ and the PE eigenvalue dependence $\mu(\delta)$ follows from

$$
\int_{0}^{x_{0}} \sqrt{\frac{\delta e^{-\kappa x^{\prime}}}{6 a^{3} \kappa}-\frac{\mu}{b}} \mathrm{~d} x^{\prime}=C,
$$

with the parameter $C$ given by ${ }^{10}$

$$
C \equiv 2\left|a i_{1}\right|^{3 / 2} /(3 \sqrt{6}) \approx 0.973 .
$$

The critical adsorption parameter $\delta_{c}$ follows from eqn (12) for $\mu=0$ that, by definition, separates bound and unbound states of the PE chain. This transition implies that the distribution of adsorbed PEs near the interface becomes infinitely wide, and the point where the oscillating and decaying solution match moves to infinity, $x_{0} \rightarrow \infty$. This yields the scaling law

$$
\delta_{c}=\frac{6 C^{2}}{4}(\kappa a)^{3} \approx 1.42(\kappa a)^{3},
$$

which agrees within just $2 \%$ with the result of the exact enumeration $^{5}$ that gives $\delta_{c}=\frac{j_{0,1}^{2}}{4}(\kappa a)^{3} \approx 1.446(\kappa a)^{3}$, see Fig. 1 . Here $j_{0,1} \approx 2.405$ is the first zero of the Bessel function $J_{0}$.

Eqn (14) illustrates the simple physical fact that, upon addition of salt, higher surface charge densities are required to stimulate PE adsorption. Fig. 1 clearly shows that larger critical adsorption parameters are necessary to trigger $\mathrm{PE}$ adsorption onto curved convex surfaces of cylinders and 


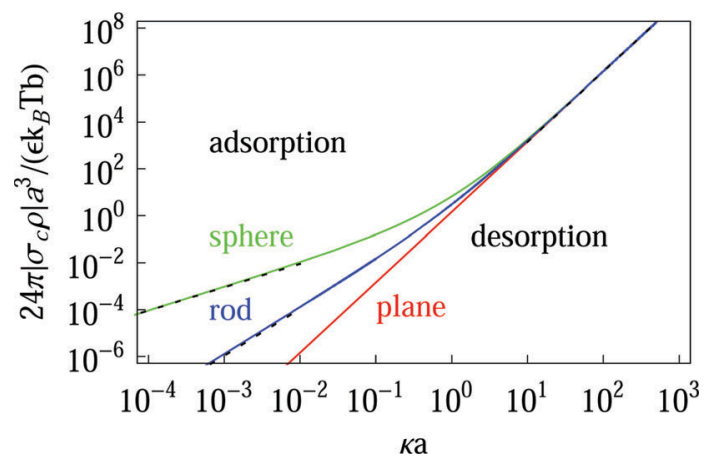

Fig. 1 Critical adsorption parameter $\delta_{c}$ obtained from the WKB approach. Scaling asymptotes (eqn (14) for the plane, eqn (18) and (22) for rod and sphere, respectively, in the limit $\kappa a \ll 1)$ are shown as dotted lines.

spheres (see the next sections), as compared to the planar situation. The effect is particular strong in the limit of low salt or large surface curvature when $\kappa a \ll 1$.

For the decaying solution, which is realized for $x>x_{0}$, we have $R>0, \xi(x)=\int_{x_{0}}^{x} \sqrt{R\left(x^{\prime}\right)} \mathrm{d} x^{\prime}$, and the monomer density distribution $P(x)$ is described by

$$
P(x) \propto\left\{\xi^{1 / 6} R^{-1 / 4} \operatorname{Ai}\left((3 \xi / 2)^{2 / 3}\right)\right\}^{2},
$$

see Fig. 2. The decaying branch of the $A i$ function satisfies automatically the condition of vanishing PE density far from the adsorbing interface.

Normalized PE density profiles have been computed for various adsorption strengths $\delta$. The width of the PE layer near the surface $w$ is evaluated at the half-height of $P(x)$. We find that far from the adsorption transition, at large adsorption strengths, it scales as $w \propto \delta^{-1 / 3}$, see Fig. 3. It is consistent with the theoretical prediction from the mean-field theories of PE adsorption. ${ }^{1}$ As one could expect, more concentrated electrolyte solutions require larger $\delta$ values for $\mathrm{PE}$ adsorption to take place. Also, at the same $\delta$, the width of the PE profile increases with increasing salt concentration, see

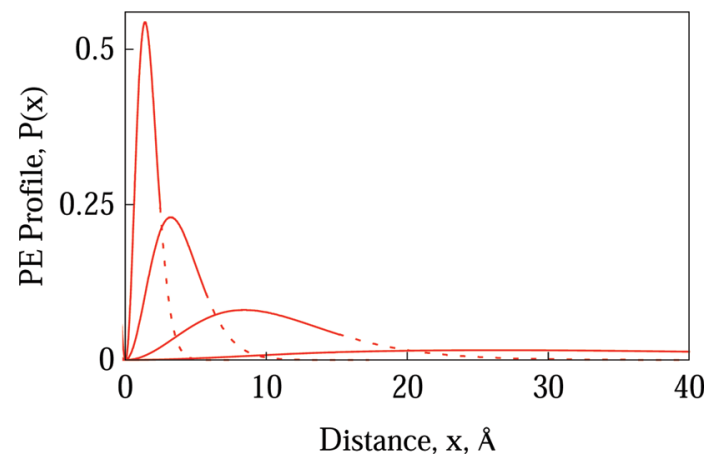

Fig. 2 Normalized PE density distributions near an adsorbing plane for $\delta=0.002,0.01,0.1,1$ (for the curves from right to left). For each curve, the solid and dotted parts represent the oscillating and decaying branch of the solution, respectively. Close to the adsorptiondesorption coexistence line, the polymer profile becomes wide, whereas far from this transition in a strong-adsorption regime the PE adsorbed layer is strongly confined near the interface. Parameters: $b=30 \AA$, $1 / \kappa=10 \AA$.

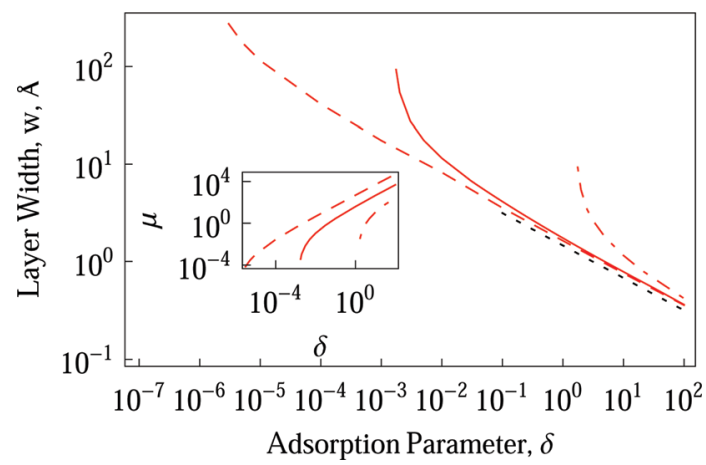

Fig. 3 PE layer thickness near the adsorbing plane at varying amount of added salt. The scaling relation $w \propto \delta^{-1 / 3}$ is shown as the black dotted line. The width diverges as $\delta \rightarrow \delta_{c}$ and for higher salinitiy this transition takes place at larger adsorption parameters. The inset shows the eigenvalues of adsorbed PEs. Parameters: $b=30 \AA, 1 / \kappa=100 \AA$ for dashed curves, $1 / \kappa=10 \AA$ for solid curves, and $1 / \kappa=1 \AA$ for dot-dashed curves.

Fig. 3. The eigenvalues of the adsorbed PE chain $\mu(\delta)$ also exhibit a strong $\delta$-dependence, as shown in the inset of this figure.

The fact that the critical adsorption transition $\delta_{c}(\kappa a)$ and the width of the adsorbed PE layer $w(\delta)$ for the planar interface is correctly reproduced, makes us confident that the WKB method can provide reliable predictions for these essential adsorption characteristics also for curved surfaces, see below.

\section{b Rod}

The equation for the radial axially-symmetric component of the Green function for PE-cylinder adsorption reads

$$
-\frac{b}{6}\left(\psi(r)_{r r}+\frac{1}{r} \psi(r)_{r}\right)-\theta \frac{K_{0}(\kappa r)}{K_{1}(\kappa a)} \psi(r)=-\mu \psi(r) .
$$

Here $K_{0,1}$ are modified Bessel functions that naturally arise when solving the linear Poisson-Boltzmann equation for the electrostatic potential near a uniformly charged rod of radius $a<r{ }^{33,34}$ After the substitution $\kappa r=u=e^{\omega}$, this equation can be rewritten in a form required by the WKB method, namely $\psi_{\omega \omega}-R \psi=0$, with

$$
R(\omega)=\frac{6 e^{2 \omega}}{b \kappa^{2}}\left(\mu-\frac{\delta b K_{0}\left(e^{\omega}\right)}{6 a^{3} \kappa K_{1}(\kappa a)}\right) .
$$

The point $r_{0}$ of zero potential is found via solving $K_{0}\left(\kappa r_{0}\right)=\frac{6 a^{3} \kappa \mu K_{1}(\kappa a)}{\delta b}$.

For the oscillating solution at $a<r<r_{0}$, we get $\xi(r)=\frac{1}{\kappa} \int_{\kappa r}^{\kappa r_{0}} \mathrm{~d} u \sqrt{\frac{\delta K_{0}(u)}{a^{3} \kappa K_{1}(\kappa a)}-\frac{6 \mu}{b}}$ that defines the $\mu(\delta)$ dependence via $\frac{1}{\kappa} \int_{\kappa a}^{\kappa r_{0}} \mathrm{~d} u \sqrt{\frac{\delta K_{0}(u)}{6 a^{3} \kappa K_{1}(\kappa a)}-\frac{\mu}{b}}=C$, with $C$ given in eqn (13). The critical adsorption condition at $\mu=0$ then follows from numerical integration of the equation

$$
\sqrt{\frac{\delta_{c}}{a^{3} \kappa^{3} K_{1}(\kappa a)}} \int_{\kappa a}^{\infty} \mathrm{d} u \sqrt{K_{0}(u)}=C \sqrt{6} .
$$

It reveals a transition from planar-like $\delta_{\mathrm{c}} \propto(\kappa a)^{3}$ scaling at large $\kappa a$ values to the scaling law $\delta_{\mathrm{c}} \propto(\kappa a)^{2}$ for $\kappa a \ll 1$, see Fig. 1. The latter scaling behavior can be obtained analytically 


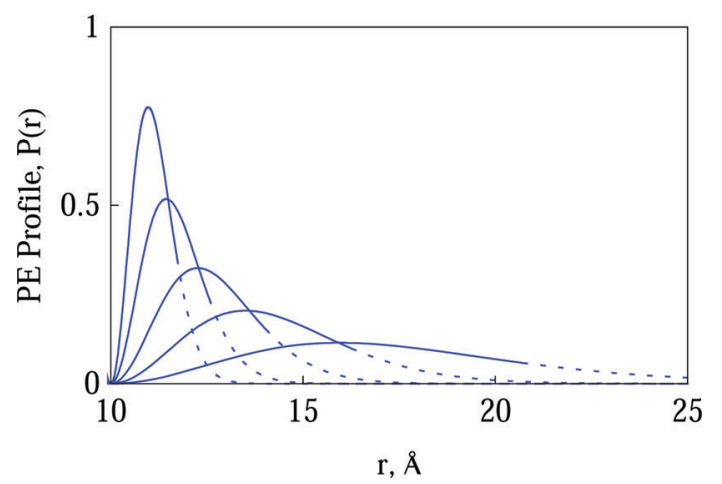

Fig. 4 Normalized PE density profiles for PE-rod adsorption. The parameters are $\delta=30,100,300,1000,3000$ (from right to left), $a=10 \AA, b=30 \AA$, and $1 / \kappa=10 \AA$.

from eqn (17) using the expansions of Bessel functions for small and large arguments (for $K_{0}(\kappa a)$ and $K_{1}(\kappa r)$, respectively). It yields the following asymptotic behavior in terms of the gamma function $\Gamma$

$$
\delta_{c} \approx \frac{6 C^{2}(\kappa a)^{2}}{\left[(\pi / 2)^{1 / 4} \Gamma(3 / 4) 2^{3 / 4}\right]^{2}} \approx 1.067(\kappa a)^{2} .
$$

The probability distribution function of PE monomers is (see Fig. 4)

$$
P(r) \propto 2 \pi r\left\{\xi^{1 / 6}(-R)^{-1 / 4} A i\left(-(3 \xi / 2)^{2 / 3}\right)\right\}^{2} .
$$

For the decaying solution at $r>r_{0}$, we define $\xi(r)=\frac{1}{\kappa} \int_{\kappa r_{0}}^{\kappa r} \mathrm{~d} u \sqrt{-\frac{\delta K_{0}(u)}{a^{3} \kappa K_{1}(\kappa a)}+\frac{6 \mu}{b}}$. Far from the point $r_{0}$ of zero potential, the expansion of the Airy function yields a simple form for the PE profile

$$
\psi(r) \propto(6 \mu / b)^{-1 / 4} r^{-1 / 2} e^{-r \sqrt{6 \mu / b}} .
$$

We emphasize a strong dependence of the decay length of this function on the PE energy level $\mu$, see also Discussion. The PE density profile is described by

$$
P(r) \propto 2 \pi r\left\{\xi^{1 / 6} R^{-1 / 4} A i(3 \xi / 2)^{2 / 3}\right\}^{2} .
$$

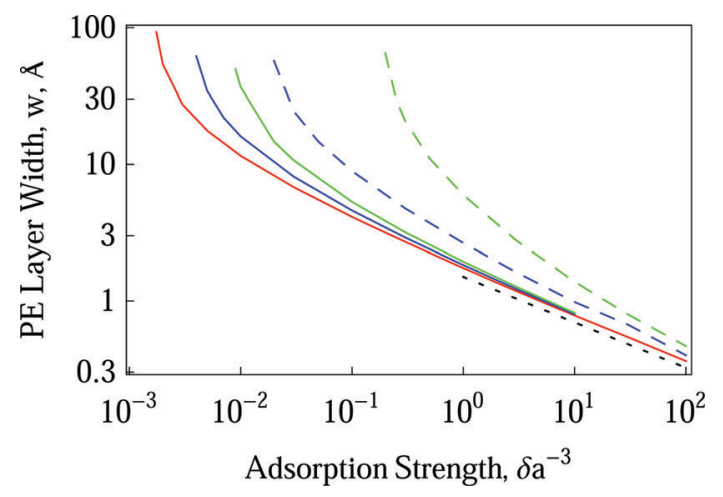

Fig. 5 Thickness $w$ of the PE layer near a planar (red), cylindrical (blue), and spherical (green) adsorbing interface. The scaling relation $w \propto \delta^{-1 / 3}$ is indicated by the black dotted line. Parameters: $b=30 \AA$, $1 / \kappa=10 \AA$, and $a=1 \AA$ for the dashed, and $a=10 \AA$ for the solid curves (for the rod and sphere cases).
The width of the adsorbed PE layer follows, again far from the adsorption transition, a $w \propto \delta^{-1 / 3}$ scaling law, as shown in Fig. 5. We emphasize here that this scaling law is to be taken with caution for all three adsorbing geometries because it is approached for very large adsorption strengths only. At such conditions, the spatial extension of very dense PE adsorbed layers can be influences by the presence of additional specific intra-chain interactions neglected in the current model.

\section{c Sphere}

The radial centrally-symmetric component of the Green function for PE-sphere electrostatic adsorption in the DebyeHückel potential satisfies the equation

$$
\begin{aligned}
& -\frac{b}{6}\left(\psi(r)_{r r}+\frac{2}{r} \psi(r)_{r}\right) \\
& -\theta a^{2} \kappa \frac{e^{\kappa a}}{1+\kappa a} \frac{e^{-\kappa r}}{r} \psi(r)=-\mu \psi(r) .
\end{aligned}
$$

In terms of a new function $\varphi=r \psi$ and new variable $u=\kappa r$, one gets $\varphi_{r r}-R(\kappa r) \varphi=0$ with the potential

$$
R(u)=\frac{6 \mu}{b}-\frac{\delta \kappa}{a} \frac{e^{\kappa a}}{1+\kappa a} \frac{e^{-u}}{u} .
$$

The point of zero potential $r_{0}$ is defined by $\frac{e^{-\kappa r_{0}}}{r_{0}}=\frac{6 \mu a(1+\kappa a)}{b \delta e^{\kappa a}}$.

For the oscillating solution at $a<r<r_{0}$, we have $\xi(r)=$ $\frac{1}{\kappa} \int_{\kappa r}^{\kappa r_{0}} \mathrm{~d} u \sqrt{-R(u)}$ and $\frac{1}{\kappa} \int_{\kappa a}^{\kappa r_{0}} \mathrm{~d} u \sqrt{-R(u) / 6}=C$ that prescribes the $\mu(\delta)$ dependence. The latter defines the critical adsorption condition (at $\mu=0$ and $r_{0} \rightarrow \infty$ ) as

$$
\delta_{c}=\frac{6 \kappa a(1+\kappa a) C^{2}}{2 \pi e^{\kappa a} \operatorname{Erf} c^{2}(\sqrt{\kappa a / 2})} .
$$

Here $\operatorname{Erfc}(y)=1-\operatorname{Erf}(y)$ is the complementary error function. Using its expansions for small and large arguments, respectively, we find for $\kappa a \ll 1$ the scaling relation

$$
\delta_{c} \approx \frac{6 C^{2}}{2 \pi} \kappa a \approx 0.904(\kappa a)^{1},
$$

and the $\delta_{c} \approx \frac{6 C^{2}}{4}(\kappa a)^{3}$ scaling for $\kappa a \gg 1$. The first relation is quite close to the $\delta_{c} \approx \kappa a$ scaling predicted from the approximate analytical solution of the PE-sphere adsorption problem derived in the Hulthen PE-sphere attractive potential, ${ }^{13}$ rather than in the

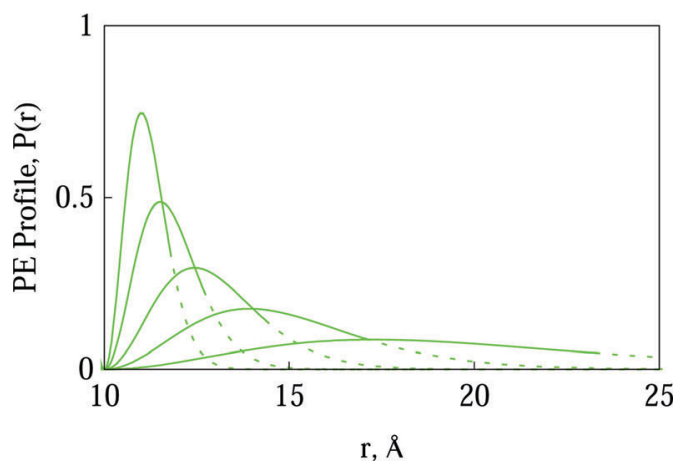

Fig. 6 PE density profiles near the oppositely charged sphere, presented for the same $\delta, \kappa, b$, and $a$ values as for PE-rod adsorption in Fig. 4. 
Yukawa-like $e^{-\kappa r} / r$-potential. The second limit is the WKB result for the plane, eqn (14). The PE density profile is $P(r) \propto 4 \pi r^{2}\left\{r^{-1} \xi^{1 / 6}(-R)^{-1 / 4} A i\left(-(3 \xi / 2)^{2 / 3}\right)\right\}^{2}$, see Fig. 6. For the decaying solution, beyond the turning point $r>r_{0}$, we have $\xi(r)=\frac{1}{\kappa} \int \mathrm{d} u \sqrt{R(u)}$ and $P(r) \propto 4 \pi r^{2}\left\{r^{-1} \xi^{1 / 6} R^{-1 / 4}\right.$ $\left.\operatorname{Ai}\left((3 \xi / 2)^{2 / 3}\right)\right\}^{2}$. $^{k r_{0}}$

Similarly to the planar and cylindrical surfaces, the width of the tightly-adsorbed PE layer near a sphere follows the law $w \propto \delta^{-1 / 3}$, see Fig. 5, in good agreement with the approximate prediction of $w \propto \delta^{-3 / 8}$ from ref. 14 and $w \propto \delta^{-2 / 5}$ obtained in ref. 15 .

\section{Discussion and conclusions}

\section{a Main effects}

Within a simple model, we determined the distinct scaling behavior for the critical surface charge density required to trigger adsorption of flexible PE chains for three fundamental geometries as a function of the Debye screening length $1 / \kappa$. The main conclusion is that the critical adsorption parameter scales as $\delta_{c} \propto\left|\rho \sigma_{c}\right| \propto \kappa^{3}$ for a planar interface, whereas for cylindrical and spherical surfaces in the large-curvature or low-salt limit we find the relations $\delta_{c} \propto(\kappa a)^{2}$ and $\delta_{c} \propto(\kappa a)^{1}$, correspondingly, see Fig. 7. Generally, as the radius of surface curvature increases and the parameter $\kappa a$ grows, a transition takes place from the large curvature limits to the planar limit. Both, for the adsorbing cylinder and sphere, this change in scaling laws takes place at $\kappa a \sim 1$, i.e., when the radius of surface curvature becomes comparable to the Debye screening length, see Fig. 1. This is another physical message of this study. For PE-rod adsorption, the transition to planar limit occurs at somewhat smaller $\kappa a$ values than for the PE-sphere situation.

The main advantage of the WKB method proposed is a unified approach for rationalizing the properties of $\mathrm{PE}$ adsorption onto oppositely charged interfaces of all three basic geometries on the same level of approximations. From the calculated relations $\delta_{c}(\kappa a)$, the corresponding critical surface charge density, critical adsorption temperature, and critical curvature radius of the surface (for rod and sphere cases) can be determined, see ref. 15 .

For a fixed $\kappa a$ value, the critical surface charge density required to initiate PE-sphere adsorption is always larger than that for PE-rod adsorption. Physically, as one moves from a plane to a rod and to a sphere, the entropic penalty of PE

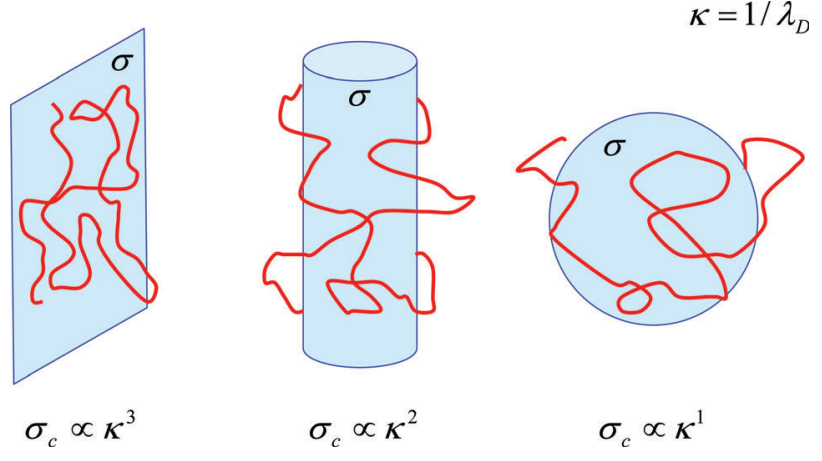

Fig. 7 A pictorial representation of WKB results for critical adsorption conditions in the low-salt limit. confinement near the surface is likely to grow. The corresponding $\delta$ values and surface charge densities to initiate the PE adsorption increase as well, see Fig. 1. Another, energy-based explanation is that a spherical surface offers less contact area ${ }^{35}$ and thus larger surface charge densities are necessary for PE adsorption, as compared to PE-rod and PE-plane complexation.

In the limit of $\kappa a \ll 1$, the reduction in the number of translational degrees of freedom for adsorbed PE chains appears to be coupled to the power of $\delta_{c} \propto(\kappa a)^{p+1}$. Namely, $p=2$ for PEs on a plane with possible chain translations in 2D, $p=1$ for PEs adsorbed on a rod with possible translations along the cylinder axis, while $p=0$ for the PE-sphere adsorption, with no chain translational freedom at all. In the opposite limit of $\kappa a \gg 1$, the scaling relations for PE-rod and PE-sphere adsorption turn into the known asymptotic behavior for a planar surface, well reproduced by the WKB analysis, see Fig. 1.

It is also interesting to note that such a regular decrease in the power of $\kappa$ resembles the change in the laws of electrostatic repulsion between the particles of corresponding geometry in simple-salt solutions. Namely, at large inter-particle separations $\kappa R \gg 1$, two uniformly charged planes, rods, and spheres repel each other following respectively the dependences $\propto e^{-\kappa R}, \propto e^{-\kappa R}(\kappa R)^{-1 / 2}$, and $\propto e^{-\kappa R}(\kappa R)^{-1} \cdot 36$

\section{b Comparison with other theories and experimental data}

Let us now compare our findings with the results of existing theories of weak PE adsorption onto curved surfaces. One well-established seminal study is the investigation of M. Muthukumar ${ }^{12}$ that employs a specific set of trial functions to mimic the peak of adsorbed PEs near the interface. For PE-rod adsorption, this method yields very good quantitative agreement with the WKB result for the critical adsorption condition $\delta_{c}$. For PE-sphere adsorption, in contrast, its outcomes disagree with the scaling predictions from the WKB model presented above as well as from the approximate solution of PE-sphere adsorption problem in the Hulthen potential derived in ref. 13. Namely, in the limit of small $\kappa a$, Muthukumar's model predicts a $\delta_{c} \propto(\kappa a)^{2}$ dependence, both for the rod and sphere situations. Note that this variational technique employs a specific dependence of trial functions on the PE eigenvalues.

The salt effects on PE complexation with oppositely charged objects have been examined in a number of excellent experimental studies, see Introduction. In particular, the complexation behavior of various PEs with oppositely charged cylindrical and spherical DMDAO micelles have been studied extensively in the group of P. Dubin, see Introduction and also the overview in Sec. 3c of ref. 14. The micelle surface charge density was controlled in experiments via a $\mathrm{pH}$-dependent protonation of surface charges. The critical charge density $\sigma_{c}$, sufficient to trigger the PE-micelle adsorption and subsequent precipitation of PE-micelle complexes from solution, has been extracted from the measurements. The results at varying solution salinity exhibit a scaling law $\sigma_{c} \propto$ $\kappa^{\nu_{1}}$ with $\nu_{1}=0.9-1.4$ for spherical micelles and $\sigma_{c} \propto \kappa^{\nu_{2}}$ with $\nu_{2}=1.8-2.5$ for the micelles of elongated, rod-like shape. ${ }^{37}$

This, by about $\kappa^{1}$, stronger dependence of $\sigma_{c}$ on salt concentration for cylindrical particles is consistent with our 
general analytical predictions in the limit $\kappa a \ll 1$, see Fig. 1. In the experimentally-relevant range of parameters, at $\kappa a=1-10$, the WKB scaling law for a cylinder is still noticeably different from that for a sphere. The absolute values of the exponents in this range approach, however, the planar limit $\delta_{c} \propto(\kappa a)^{3}$. This discrepancy with experimental values can be resolved when using the concept of saltdependent electrostatic persistence length. The latter is often approximated for flexible PEs by $b_{e l} \propto \kappa^{-1} \cdot{ }^{38,39}$ As a rough estimate, the scaling exponent for the critical value of $\left|\rho \sigma_{c}\right|$ as function of $\kappa$ is then reduced by about 1 that brings the value in the experimental range of $\nu_{1,2}$. Some additional effects of chain persistence length on the $\nu$ value might also arise from a preferential PE adsorption along the rod axis.

\section{c Possible extensions and perspectives}

We hope that the current investigation will improve the understanding of fundamental properties of PE electrostaticallydriven adsorption onto curved interfaces, and will stimulate new experimental studies. Several directions for future extensions of the model are apparent. One of them is the consideration of finite-length chains, ${ }^{40,41}$ instead of infinitely long PEs, to be able to directly compare the theoretical results with the outcomes of experiments and computer simulations, ${ }^{42-44}$ see also refs. 45 and 46 . In this case, the calculations become however more cumbersome, because for shorter polymers the eigenvalues for the excited states start to make a progressively larger contribution to the eigenfunction expansion.

We expect that our theoretical results for critical adsorption can be directly compared with coarse-grained computer simulations, which exploit the Debye-Hückel PE-surface potential (the electrostatic energy of the monomer-surface attraction is $<k_{\mathrm{B}} T$ ). In the weak adsorption limit, when the conformational properties of polymers are only slightly perturbed, we expect the actual PE statistical properties (affected by, e.g., excluded volume interactions) to have a rather small affect on the critical adsorption behavior. This is confirmed in ref. 13, by the agreement between the critical PE-sphere adsorption radius obtained from simulations and predicted by theory. However, for PE chains strongly adsorbed on surfaces, far from the adsorption-desorption transition, the self-interactions within the PE chain as well as the counterion release accompanied by a corresponding entropy gain, are likely to contribute significantly to the adsorption properties.

Another ingredient for a more realistic description of PE adsorption is polymer persistence, beyond the limit of Gaussian chains. Upon adsorption onto curved surfaces such as the sphere, the polymers unavoidably bend that has a strong effect on the adsorption characteristics. It is particularly important when the polymer segment length $b$ value becomes comparable to the surface radius of curvature (larger $\sigma_{c}$ are necessary for adsorption of stiffer chains). This limit requires a self-consistent theory of equilibrium adsorption of semiflexible PEs from solution onto an attractive sphere. A simpler situation of irreversible strong PE-sphere adsorption for highly charged PEs have been thoroughly analyzed in a number of theoretical studies. ${ }^{14,47-49}$
We want to mention that in the limit of weak adsorption, the radial distribution function $P(r)$ does not provide any information about the polymer ordering near the adsorbing rod or sphere. The situation changes in the strong adsorption limit, when the chain fluctuations can be neglected and a static pattern of ordered PEs emerges on the rod/sphere surface. For strong PE-rod adsorption, a polymer ordering and helical-like wrapping have been quantified in a number of theoretical studies. ${ }^{50-52}$ These results might find their applications in the cutting edge area of DNA interactions with carbon nanotubes. For the latter, a helical wrapping of flexible singlestranded DNA chains around single-wall carbon nano-tubes with a well-defined helical pitch has recently been systematically examined in experiments, ${ }^{53-55}$ quantified by theoretical modeling, ${ }^{56-58}$ and mimicked by computer simulations. ${ }^{59-61}$

In addition, PE chains weakly adsorbed at a charged interface might encounter other electrostatic effects neglected in this study. One of them is a patchiness of charges on the surface ${ }^{62-65}$ and non-uniform charge distributions on PE chains ${ }^{66,67}$ that both can affect the adsorption-desorption equilibrium. Another feature is the electrostatic image force ${ }^{68-71}$ encountered by charges on PE chains deposited next to a low-dielectric interface. This generates a short-range PE-surface repulsion (for a plane, e.g., the image forces have half as short screening length compared to direct Debye-Hückel PE-surface attraction). The adsorbed PEs are thus effectively "pushed aside" from the surface $^{72}$ and generally larger surface charge densities are required for PE-surface adsorption. ${ }^{73}$ One biological application of such image-force effects is the complexation of flexible single-stranded nucleic acid chains on the interior of capsid shells of many single-stranded RNA/DNA viruses, ${ }^{74,75}$ both of spherical and filamentous appearance. Within the WKB approach, the image forces can be included in the analysis via a straightforward modification of the potential function $R$.

The WKB method can also provide a rational description of $\mathrm{PE}$ adsorption in the presence of some non-electrostatic short-range PE-surface attraction ${ }^{76}$ that could be modelled as an additional square-well potential near the surface. ${ }^{77,78}$ All these interesting and motivating features are, however, beyond the scope of this study. Their potential impact on the scaling characteristics of the PE critical adsorption transition and the thickness of the PE adsorbed layers in the three fundamental adsorption geometries is yet to be quantified in future theoretical studies.

\section{Acknowledgements}

The work was partly supported by the DFG via a grant to AGC. Scientific discussions with P. Dubin, M. Fedorov, and S. Rotkin, as well as the comments of anonymous reviewers are gratefully acknowledged.

\section{References}

1 R. R. Netz and D. Andelman, Phys. Rep., 2003, 380, 1.

2 A. V. Dobrynin and M. Rubinstein, Prog. Polym. Sci., 2005, 30, 1049 .

3 P. A. Pincus, et al., J. Phys., 1984, 45, 725.

4 E. Gurovitch and P. Sens, Phys. Rev. Lett., 1999, 82, 339. 
5 F. W. Wiegel, J. Phys. A: Math. Gen., 1977, 10, 299.

6 M. Muthukumar, J. Chem. Phys., 1987, 86, 7230.

7 R. R. Netz and J. F. Joanny, Macromolecules, 1999, 32, 9026.

8 R. Messina, J. Phys.: Condens. Matter, 2009, 21, 113102.

9 X. Man and D. Yan, Macromolecules, 2010, 43, 2582.

10 T. Odijk, Macromolecules, 1980, 13, 1542.

11 T. Odijk, Langmuir, 1991, 7, 1991.

12 F. van Goeler and M. Muthukumar, J. Chem. Phys., 1994, 100, 7796.

13 R. G. Winkler and A. G. Cherstvy, Phys. Rev. Lett., 2006, 96, 066103.

14 A. G. Cherstvy and R. G. Winkler, J. Chem. Phys., 2006, 125, 064904.

15 R. G. Winkler and A. G. Cherstvy, J. Phys. Chem. B, 2007, 111, 8486.

16 T. Wallin and P. Linse, J. Phys. Chem., 1996, 100, 17873.

17 P. Chodanowski and S. Stoll, J. Chem. Phys., 2001, 115, 4951.

18 C. Y. Kong and M. Muthukumar, J. Chem. Phys., 1998, 109, 1522.

19 P. Welch and M. Muthukumar, Macromolecules, 2000, 33, 6159.

20 R. Messina, C. Holm and K. Kremer, J. Polym. Sci., Part B: Polym. Phys., 2004, 42, 3557.

21 S. Stoll and P. Chodanowski, Macromolecules, 2002, 35, 9556.

22 D. W. McQuigg, et al., J. Phys. Chem., 1992, 96, 1973.

23 C. L. Cooper, et al., Biomacromolecules, 2006, 7, 1025.

24 A. B. Kayitmazer, et al., J. Phys. Chem. B, 2003, 107, 8158.

25 C. L. Cooper, et al., Curr. Opin. Colloid Interface Sci., 2005, 10, 52.

26 A. B. Kayitmazer, et al., Biomacromolecules, 2010, 11, 3325.

27 M. Antonov, M. Mazzawi and P. L. Dubin, Biomacromolecules, 2010, 11, 51.

28 R. Podgornik, J. Phys. Chem., 1992, 96, 884.

29 I. Borukhov, D. Andelman and H. Orland, J. Phys. Chem. B, 1999, 103, 5042.

30 R. E. Langer, Phys. Rev., 1937, 51, 669.

31 M. V. Berry and K. E. Mount, Rep. Prog. Phys., 1972, 35, 315.

32 I. S. Gradshteyn and I. M. Ryzhik, "Table of Integrals, Series, and Products", Academic Press, 1980.

33 A. G. Cherstvy and A. A. Kornyshev, J. Phys. Chem. B, 2005, 109, 13024.

34 A. G. Cherstvy, J. Phys. Chem. B, 2008, 112, 12585.

35 Y. Wang, P. L. Dubin and H. Zhang, Langmuir, 2001, 17, 1670.

36 V. A. Parsegian, Annu. Rev. Biophys. Bioeng., 1973, 2, 221.

37 X. H. Feng, et al., Macromolecules, 2001, 34, 6373.

38 R. Everaers, A. Milchev and V. Yamakov, Eur. Phys. J. E, 2002, 8, 3.

39 A. V. Dobrynin, Macromolecules, 2005, 38, 9304.

40 X. Man, et al., Macromolecules, 2008, 41, 5451.

41 N. Kaellrot and P. Linse, J. Phys. Chem. B, 2010, 114, 3741.
42 A. V. Dobrynin, Curr. Opin. Colloid Interface Sci., 2008, 13, 376.

43 S. J. de Carvalho, Europhys. Lett., 2010, 92, 18001.

44 S. V. Larin, et al., J. Phys. Chem. B, 2010, 114, 2910.

45 M. V. Fedorov, et al., J. Am. Chem. Soc., 2009, 131, 10854.

46 M. V. Fedorov, et al., Chem. Commun., 2009, 896.

47 K.-K. Kunze and R. R. Netz, Phys. Rev. Lett., 2000, 85, 4389.

48 H. Boroudjerdi and R. R. Netz, J. Phys.: Condens. Matter, 2005, 17, S1137.

49 S. Ulrich, et al., Curr. Opin. Colloid Interface Sci., 2006, 11, 268.

50 K.-K. Kunze and R. R. Netz, Europhys. Lett., 2002, 58, 299.

51 A. G. Cherstvy and R. G. Winkler, J. Chem. Phys., 2004, 120, 9394.

52 F. J. Solis, et al., Soft Matter, 2011, 7, 1456.

$53 \mathrm{X}$. Tu, et al., Nature, 2009, 460, 250.

54 X. Tu and M. Zheng, Nano Res., 2008, 1, 185.

55 D. A. Yarotski, et al., Nano Lett., 2009, 9, 12.

56 S. Manohar, et al., J. Phys. Chem. C, 2007, 111, 17835.

57 I. Gurevitch and S. Srebnik, J. Chem. Phys., 2008, 128, 144901.

58 S. V. Rotkin, Annu. Rev. Phys. Chem., 2010, 61, 241.

59 R. J. Johnson, et al., Nano Lett., 2009, 9, 537.

60 T. Vogel and M. Bachmann, Phys. Rev. Lett., 2010, 104, 198302.

61 D. Roxbury, S. Manohar and A. Jagota, J. Phys. Chem. C, 2010, 114, 13267.

62 M. Muthukumar, J. Chem. Phys., 1995, 103, 4723.

63 J. McNamara, C. Y. Kong and M. Muthukumar, J. Chem. Phys., $2002, \mathbf{1 1 7}, 5354$

64 N. Hoda and S. Kumar, J. Chem. Phys., 2008, 128, 124907.

65 E. Bianchi, R. Blaak and C. N. Likos, Phys. Chem. Chem. Phys., 2011, 13, 6397.

66 R. Messina, Eur. Phys. J. E, 2007, 22, 325.

67 S. Ulrich, et al., Macromol., 2011, 44, 1661.

68 M. Kanduc and R. Podgornik, Eur. Phys. J. E, 2007, 23, 265.

69 R. Messina, Phys. Rev. E, 2004, 70, 051802.

70 M. Manciu and E. Ruckenstein, Adv. Colloid Interface Sci., 2003, $105,63$.

71 M. Seijo, et al., Phys. Chem. Chem. Phys., 2006, 8, 5679.

72 M. Seijo, et al., J. Chem. Phys., 2009, 131, 174704.

73 A. G. Cherstvy, work in preparation.

74 V. A. Belyi and M. Muthukumar, Proc. Natl. Acad. Sci. U. S. A., 2006, 103, 17174

75 A. G. Cherstvy, Phys. Chem. Chem. Phys., 2011, DOI: 10.1039/ c0cp02796k.

76 J. M. Y. Carrillo and A. V. Dobrynin, Langmuir, 2007, 23, 2472.

77 T. M. Birshtein and O. V. Borisov, Polymer, 1991, 32, 916.

78 A. I. Chervanyov and G. Heinrich, J. Chem. Phys., 2009, 131, 104905. 